\title{
Ovine panel reactive antibody assay of HLA responsivity to allograft bioengineered vascular scaffolds
}

\author{
Ara Ketchedjian, $\mathrm{MD}^{\mathrm{a}}$ \\ Paula Kreuger, BS $^{\mathrm{a}}$ \\ Howard Lukoff, BS ${ }^{a}$ \\ Elliot Robinson, $\mathrm{BS}^{\mathrm{a}}$ \\ Alyce Linthurst-Jones, $\mathrm{MS}^{\mathrm{b}}$ \\ Katrina Crouch, BS ${ }^{\mathrm{b}}$ \\ Lloyd Wolfinbarger, $\mathrm{PhD}^{\mathrm{b}}$ \\ Richard Hopkins, MDa
}

From the Division of Cardiothoracic Surgery and the Collis Surgical Research Laboratory, ${ }^{\text {a }}$ Brown University, Providence, RI, and Lifenet, ${ }^{\mathrm{b}}$ Virginia Beach, Va.

Supported by grants from the Children's Heart Foundation, Collis Cardiothoracic Laboratory, Roddy Scholarship, and Medtronic Corporation. Lifenet Tissue Services provided the decellularization technology.

Read at the Georgia Institute of Technology Workshop, Hilton Head, SC, March 10, 2004.

Received for publication March 15, 2004; revisions received June 15, 2004; accepted for publication June 22, 2004.

Address for reprints: Richard A. Hopkins, MD, Department of Cardiothoracic Surgery, Rhode Island Hospital, Brown University, 500 MOC, 2 Dudley St, Providence, RI 02905 (E-mail: rahopkins@ lifespan.org).

J Thorac Cardiovasc Surg 2005;129:159-66 $0022-5223 / \$ 30.00$

Copyright $\odot 2005$ by The American Association for Thoracic Surgery

doi:10.1016/j.jtcvs.2004.06.017
Background: Increasing evidence implicates immune response as a contributing factor in the failure of allograft valve transplants. Increases in panel reactive antibodies have been identified in human subjects. To correlate these responses with novel preimplantation processing methods to reduce cellularity, both a relevant panel reactive antibody assay and a chronic implantation animal model are necessary. We modified a human flow cytometric panel reactive antibody assay for ovine model use to detect antibody responses to residual antigen-loading decellularized scaffolds engineered from pulmonary artery tissue.

Methods: A clinical panel reactive antibody assay was modified with anti-sheep antibodies. Dimethyl sulfoxide cryopreserved $(n=4)$ and decellularized scaffolds $(n=8)$ fashioned as patches from pulmonary arteries were implanted for study. Fresh (nonprocessed) tissue implants were used as positive controls $(\mathrm{n}=2)$, and sham-treated animals were used as negative controls $(n=2)$. Baseline, 10-week, and 20-week blood samples were assayed for panel reactive antibody levels. Immunohistochemistry with anti-major histocompatibility complex antibodies were performed on preimplantation scaffolds.

Results: Chronic implants of fresh tissue stimulated strong panel reactive antibody responses. Classically cryopreserved tissues provoked modest panel reactive antibody responses to major histocompatibility complex I antigen and no response to major histocompatibility complex II antigen. Decellularized tissue scaffolds provoked minimal to no panel reactive antibody responses to either major histocompatibility complex I or II antigen. Immunohistochemistry correlated with the panel reactive antibody results by identifying significant amounts of major histocompatibility complex I and II in fresh tissue, reduced antigen staining in cryopreserved control tissues, and minimal amounts in decellularized tissues.

Conclusions: These studies with an ovine modified panel reactive antibody assay confirmed minimal immune allosensitization to transplanted decellularized tissue patches. Qualifying criteria for putative tissue-engineered scaffolds should include minimal recipient panel reactive antibody response.

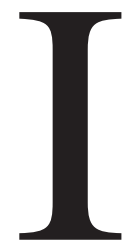

ncreasing data have implicated the body's immune response in the failure of implanted allograft cardiovascular tissues. ${ }^{1-8}$ This has been most evident in pediatric cryopreserved allograft valve reconstructions, where valves undergoing accelerated failure and functional degeneration have been explanted and found to have lymphocytic infiltration within the leaflets and conduit wall. ${ }^{9-11}$ Bioengineering technologies are being used to develop alternate implantable acellular scaffolds that are nonantigenic and thus potentially 
biologically suitable for prolonged functionality and even possible autorecellularization with autologous cells. One such alternative is decellularized vascular scaffolds fashioned from allograft aortic and pulmonary artery tissue. Such acellular scaffolds are derived from allograft vascular tissues that have been processed with detergents and enzymes to remove cells and cellular debris, presumably removing the antigens most likely to initiate allosensitization. However, to demonstrate efficacy, only human circulating panel reactive antibodies (PRAs) have been measured in response to clinically implanted tissues. A large-animal model is necessary to identify allosensitization and the effects of increased circulating HLA antibodies on implanted valvular and vascular tissue function. We have been able to modify a beaded flow cytometric PRA method to detect circulating antibodies to major histocompatibility complex (MHC) antigens in the chronic implantation sheep model. This is ideal because the chronic implantation sheep model has been strongly recommended for valvular and vascular implant tissue testing (ISO 5840:1996 and US Food and Drug Administration Replacement Heart Valve Guidance Document: 1994). In this study, cryopreserved and decellularized tissue scaffolds were implanted in juvenile sheep that were then monitored for the production of circulating HLA antibodies. Immunohistochemistry for class I and class II MHC antigens was performed on preimplantation tissues to correlate the observed PRA responses with histologically identifiable MHC I and II antigens retained in the putative scaffold test patches.

\section{Materials and Methods}

\section{Tissue Acquisition}

Ovine hearts were obtained from laboratory-grade sheep (Animal Technologies, Tyler, Tex). They were shipped in an antibiotic solution (RPMI 1640 supplemented with polymyxin B). The pulmonary valves were dissected and stored in an antibiotic solution (RPMI 1640 supplemented with polymyxin B, cefoxitin, lincomycin, and vancomycin) at $4^{\circ} \mathrm{C}$ until either cryopreservation or decellularization processing ( $24 \pm 2$ hours).

\section{Cryopreservation}

Control tissues were cryopreserved after the disinfection process was completed. The tissues were cryopreserved in RPMI 1640, $10 \%$ fetal calf serum, and $10 \%$ dimethyl sulfoxide by using a Cryomed controlled-rate freezer (Thermo Electron Corporation, Waltham, Mass). The tissues were frozen at $1^{\circ} \mathrm{C}$ per minute until they reached $-40^{\circ} \mathrm{C}$, after which they were subsequently held in vapor-phase liquid nitrogen $\left(-180^{\circ} \mathrm{c}\right)$ until implantation.

\section{Decellularization}

The decellularization was performed with a unique detergentenzyme-water decellularization process developed at LifeNet Tissue Services (US Patent No. 6,432,712). The grafts were placed in the decell flow-through chamber, and the detergent ( $N$-lauroylsarcosinate), Benzonase endonuclease solution, and polymyxin B were circulated sequentially by using a peristaltic pump for 24 to 36 hours. After decellularization, the detergent solution was drained, and sterile water was recirculated through the tissue chamber and through a bed of hydrophobic and anion exchange resins (to remove the anionic detergent) for a time equivalent to the detergent cycle.

\section{Operation}

Sixteen juvenile sheep (age range, 6-18 months) were used. All animals were acclimated for 1 week at the animal care facility. Animal handling was in accordance with Brown University institutional guidelines for the use and care of animals, and the institutional review board approved the experimental procedures. All studies also conformed to the "Guide for the Care and Use of Laboratory Animals" (National Institutes of Health publication No. 85-23, revised 1985). Anesthesia was induced with propofol (2 $\mathrm{mg} / \mathrm{kg}$ ) administered through an intravenous line placed in the leg. Once intubated, the sheep were transferred to a preparatory table and sheared. Electrocardiographic and capnographic monitors were placed. A small incision was made in the left side of the neck to allow placement of a carotid artery catheter and an internal jugular triple-lumen catheter for hemodynamic monitoring purposes. The chest was opened through a thoracotomy in the left fourth intercostal space. The venous cannula was placed in the right atrium. An arterial cannula was placed in the descending thoracic aorta. Cardiopulmonary bypass with oxygenation was initiated. After clamps were placed, surgical defects were created for implantation sequentially in the aorta and pulmonary artery. Elliptical patches fashioned from the pulmonary artery conduit were sutured to the defects with 4-0 polydioxanone sutures. Positive control tissues known to contain much antigenic cellular material (fresh, nonprocessed ovine pulmonary valves) were implanted in the descending aorta. All animals received postoperative antibiotics (amoxicillin [INN: amoxicilline], $5 \mathrm{mg} / \mathrm{kg}$ twice daily, and amikacin, $5 \mathrm{mg} / \mathrm{kg}$ twice daily) for 2 weeks and buprenorphine (1 amp twice daily) analgesia for 3 days.

Four animals per group ( $\mathrm{n}=12$ total) were implanted with patches constructed from one of three tested tissue-processing methods: cryopreservation; fresh decellularization; or cryopreservation followed by decellularization. Patches were placed in the aortic and pulmonary positions for each animal ( $n=24$ patches). Two animals in each test category were killed at the 10- and 20 -week time periods. Four additional control animals (2 shamtreated animals and 2 animals receiving fresh antigen-rich valve implants) were PRA assayed per protocol and killed at 20 weeks only.

Explantation was performed through the same thoracotomy incision after drawing a final PRA sample. Patch measurements were made under physiologic pressures. The animals were killed for tissue harvest by the administration of phenobarbital (324 $\mathrm{mg} / \mathrm{mL} ; 15 \mathrm{~mL}$ per $100 \mathrm{lb}$ ) administered through peripheral intravenous access.

\section{Flow Cytometry}

Blood specimens were collected from the jugular veins of the sheep and allowed to stand for 2 hours. Clots were removed from tubes, and remaining serum was run through a HiTrap purification system (Amersham Biosciences, Buckinghamshire, England) and 
then incubated with beads (One Lambda, Inc) tagged with 30 MHC I and 30 MHC II antigens for 30 minutes in the dark with continuous gentle shaking. The beads were washed with buffer solution and centrifuged at 11,500 rpm for 2 minutes. The supernatant was removed, and the beads were incubated with a rabbit anti-sheep fluorescein isothiocyanate antibody (Jackson ImmunoResearch, West Grove, Pa) for 30 minutes in the dark with continuous shaking. The beads were washed with buffer solution and centrifuged at 11,500 rpm for 2 minutes. Supernatant was removed, and the beads were fixed in $10 \%$ formalin and analyzed on a Becton Dickinson FACS flow cytometer (Becton Dickinson, Franklin Lakes, NJ). Human positive and negative controls were used for calibration. FL1 histograms were obtained for all specimens. Results are reported as the percentage of beads fluorescing, and increases of greater than $10 \%$ are considered positive responses (per One Lambda), as in clinical use.

\section{Histology and Immunohistochemistry}

For MHC staining, segments of the pulmonary and arterial walls containing test scaffold patches were flash-frozen with dry ice. They were stored at $-80^{\circ} \mathrm{C}$ until sectioning with a cryostat into $8-\mu \mathrm{m}$ sections, fixed with cold acetone, and stored at $-20^{\circ} \mathrm{C}$ until staining. The slides were incubated in Dako serum free protein blocking solution (Dako, Carpinteria, Calif) for 15 minutes at room temperature. After a 5-minute rinse with $\mathrm{dH}_{2} \mathrm{O}$, the slides were placed in primary antibody for 30 minutes. Mouse anti-sheep MHC I and II (VMRD, Pullman, Wash) were diluted to 1:50 in 0.1 $\mathrm{mol} / \mathrm{L}$ phosphate buffer with $5 \%$ fetal bovine serum. Slides were incubated with a secondary antibody, biotinylated mouse immunoglobulin G (Vector ABC; Vector Labs, Burlingame, Calif), for 30 minutes and then with a Vector diaminobenzidine peroxidase substrate kit until reaction (5-10 minutes). The slides were rinsed and stained with Vector hematoxylin for 15 minutes. Slides were rinsed and dehydrated through a series of alcohols and Citrisolv (Fisher Scientific, Pittsburgh, Pa) and permanently covered with a Surgipath Micromount (Surgipath Medical Industries, Richmond, IIl).

\section{Statistical Analysis}

The quantitative data are presented as a percentage of beads (as recommended for the analogous human assay by One Lambda) that are fluorescence positive for antibody binding (PRA), as determined by means of flow cytometry. Because sham control PRA values approach baseline values soon after the operation, true increases are identified by subtracting baseline values from measured explant PRA values. Group means are compared and analyzed by means of 1-way analysis of variance (ANOVA; Sigma Stat, version 2.03) and the Student-Newman-Keuls method.

\section{Results}

Immunohistochemistry staining with anti-MHC I and II antibodies (VMRD) were performed on samples of all preimplantation tissues. Nonprocessed (fresh) tissue implants demonstrated intact endothelium on hematoxylin and eosin (H\&E) staining. The endothelium stained strongly for MHC I antigen (Figure 1). The staining for MHC II antigen was slightly weaker but still present throughout the tissue (Figure 2). Cryo- preserved tissues demonstrated variable endothelial presence on H\&E staining. Although quantitatively less than nonprocessed tissues, MHC I and II antigens were identified throughout cryopreserved control tissues (Figures 3 and 4). Decellularized tissues never demonstrated endothelium or myofibroblasts on H\&E staining (Figures 5 and 6). MHC I and II staining demonstrated significantly smaller amounts of antigen within the decellularized tissues, which, when present, appeared to be localized on the adventitial aspect of the decellularized vascular tissues (Figures 7 and 8).

Flow cytometry was performed on the weekly blood specimens of both the positive and negative control animals (those receiving nonprocessed tissue implants and sham-treated animals). Results demonstrated increased levels (over baselines) of circulating PRA in animals receiving nonprocessed implants (ie, fresh and antigen rich) within 10 weeks. The 2 shamtreated animals demonstrated a gradual decrease in circulating PRA levels soon after the operation, with a gradual return toward baseline values over time (Figure 9, $A$, graphic depiction of MHC I, and Figure 9, $B$, graphic depiction of MHC II). Cryopreserved patches evoked variable levels of PRA response. One each of the two 10- and 20-week animals demonstrated a positive response. None of the four 10-week animals and only one of the four 20-week animals implanted with decellularized tissues demonstrated any positive PRA increases (Table 1). The reduced PRA response was significant when comparing decellularized with both cryopreserved and fresh tissue for MHC I and significant for MHC II when comparing decellularized and fresh tissue $(P<.05$, ANOVA for each comparison).

The decellularized scaffolds healed with minimal inflammation but with fibrous sheath formation and some recellularization with cell migration. The aortic patches healed with more extensive fibrous sheaths histologically when compared with patches in the pulmonary circulation, most likely attributed to increased shear stress. There were no aneurysms or endocarditis noted in any of the patches.

\section{Discussion}

Levels of PRA after the implantation of vascular transplant tissues have been studied in the pediatric and adult populations. Although the specific responses have varied among different studies, the overall trend has demonstrated that circulating PRA levels increase after tissue implantation as a result of allosensitization by donor antigens. ${ }^{6,12-14}$ Studies have also suggested that the implantation of decellularized tissues results in a statistically significant reduction of circulating PRA when compared with that seen in cryopreserved control tissues. ${ }^{15}$ However, these studies do have certain limitations. Because human subjects have been the sole subjects of circulating antibody studies, it has been difficult to correlate temporal changes in antibody levels to the histologic status of the tissue (eg, degeneration, inflam- 

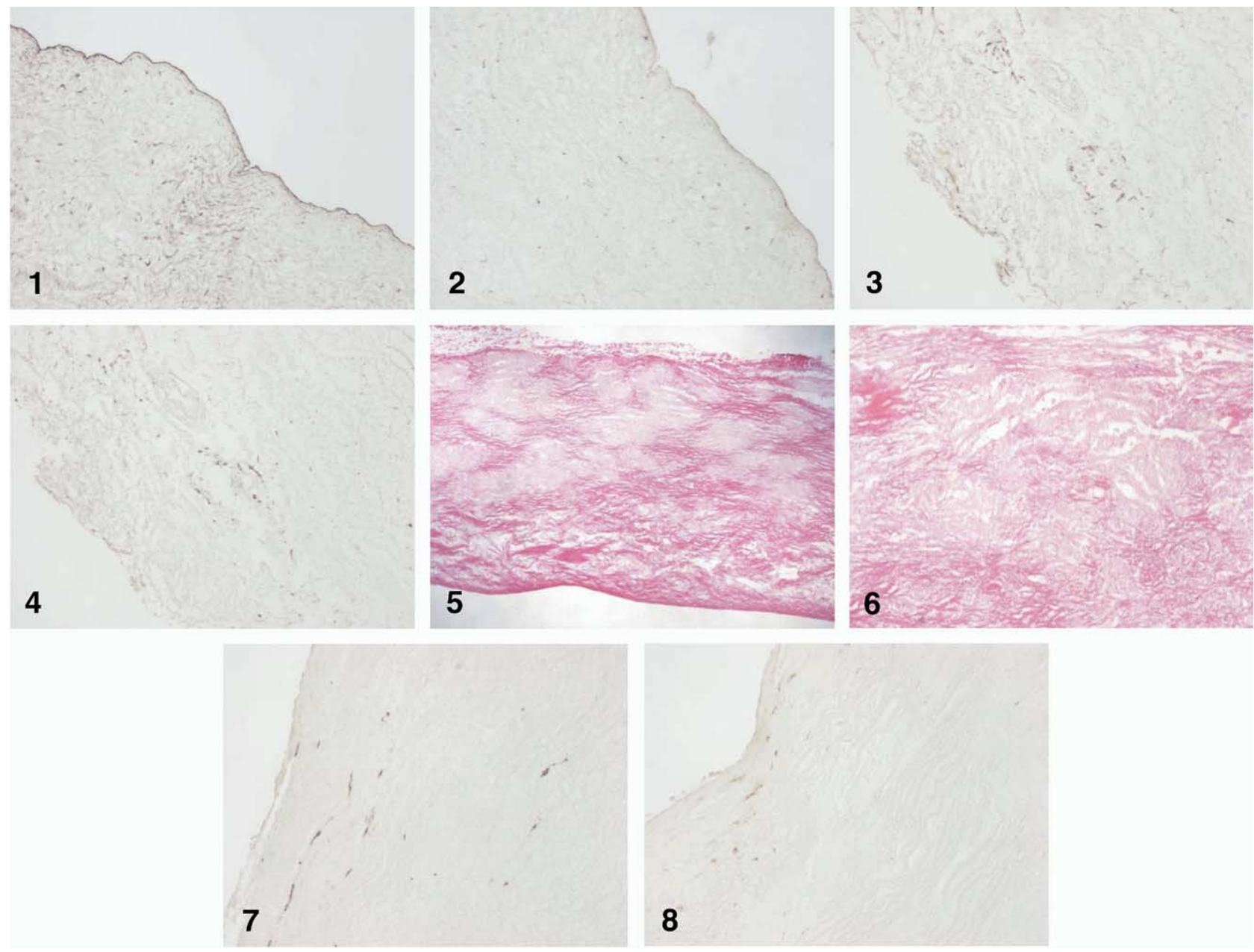

Figure 1. Immunohistochemistry staining of MHC I antigen of nonprocessed tissues. MHC I staining demonstrates antigen presence on the endothelium and diffusely throughout the tissue matrix. (Original magnification 200 $\times$.) Figure 2. Immunohistochemistry staining for MHC II antigen of nonprocessed tissue. There are only small amounts of MHC II antigen scattered throughout the tissue matrix. (Original magnification $200 \times$.)

Figure 3. Immunohistochemistry staining for MHC I antigen in cryopreserved tissue. There are scattered amounts of MHC I antigen throughout the tissue. The presence of an intact endothelium was not identified. (Original magnification $200 \times$.)

Figure 4. Immunohistochemistry staining for MHC II antigen in cryopreserved tissue. There are only small amounts of MHC II antigen throughout the tissue matrix. (Original magnification $200 \times$.)

Figure 5. H\&E-stained preimplantation decellularized tissue. There is no histologic evidence of residual intact cells or cellular debris. (Original magnification $100 \times$.)

Figure 6. High-power (H\&E) view of decellularized tissue (before implantation). There is no histologic evidence of residual intact cells or cellular debris. (Original magnification $200 \times$.)

Figure 7. Immunohistochemistry staining for MHC I antigen in decellularized tissue. The MHC I antigen is isolated in small clusters along the adventitial border. This is a worst-case example; most fields demonstrated no stainable antigenic debris. (Original magnification $200 \times$.)

Figure 8. Immunohistochemistry staining for MHC II antigen in decellularized tissue. Small amounts of MHC II antigen are present in small clusters along the adventitial aspect of the tissue. This is a worst-case example; most fields demonstrated no stainable antigenic debris. (Original magnification $200 \times$.)

mation, and antigen content). An animal model is necessary to obtain tissue for histologic evaluation at varying time points after implantation.
The primary goal of this experiment was to modify a beaded flow cytometric method to be useful for HLA antibody detection in the chronic sheep implantation model. 

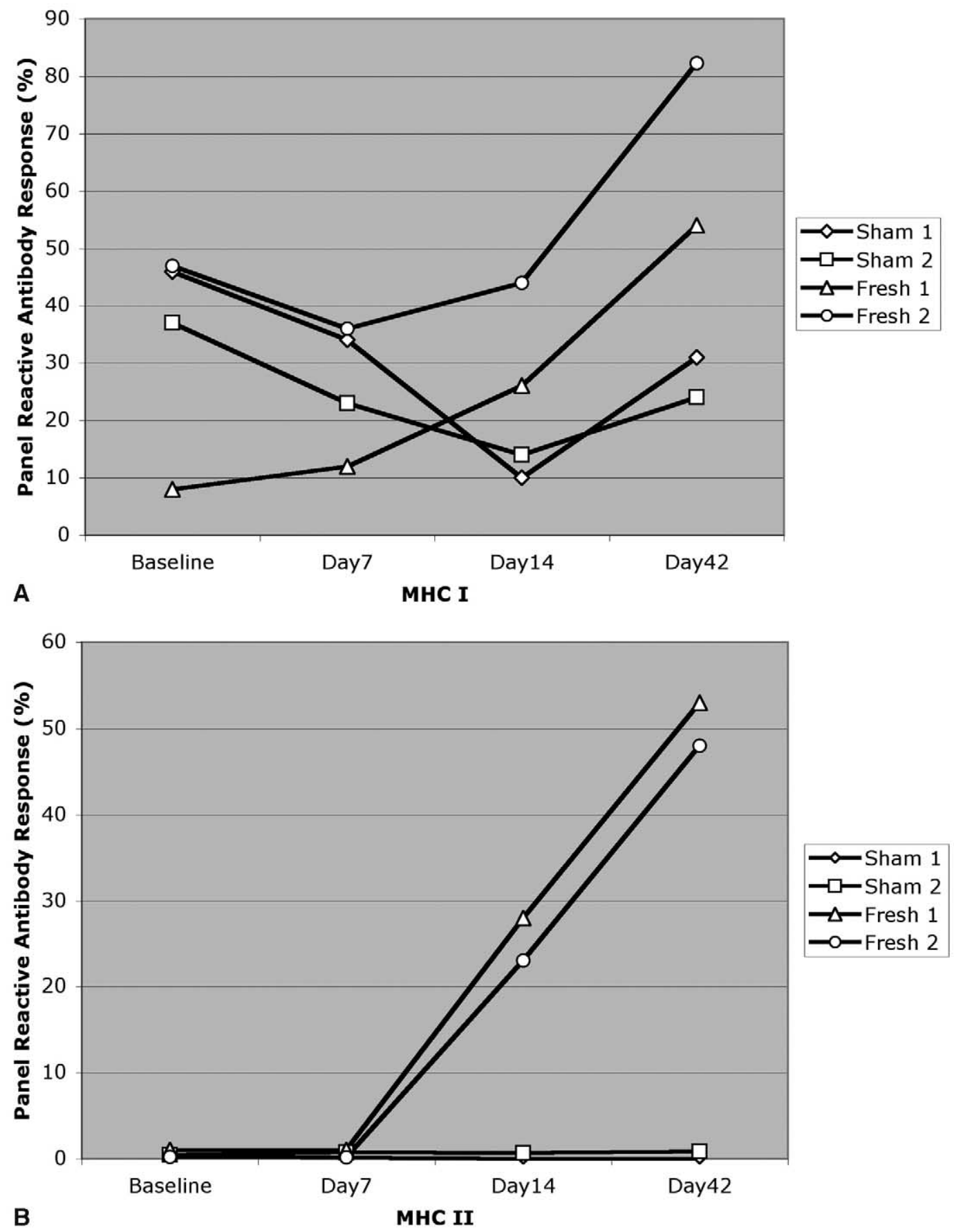

Figure 9. These graphs demonstrate circulating PRA levels determined before the operation (at baseline) and at 1 week (day 7), 2 weeks (day 14), and 6 weeks (day 42) in nonprocessed fresh (positive control tissue) and sham-treated (negative control tissue) control animals. Nonprocessed (fresh) implants demonstrated a strong increase in both MHC I (A) and II (B) PRA levels. There were no positive responses in sham-treated animals (operation without implantation). The $y$-axis shows the percentage of beads fluorescing, indicating the presence of antibodies in the recipient sheep to the tagged antigens ( $\geq 10 \%$ increases over baseline are considered positive responses).

Anti-sheep MHC antibodies were used to demonstrate recognition on the latex beads ( $99 \%$ response by means of flow cytometry). Sham-treated animals (started on cardiopulmo- nary bypass without tissue implantation) were used as negative controls and appropriately demonstrated no increase in PRA levels after surgical intervention. Nonprocessed 
TABLE 1. PRA values (indicating relative presence or absence of recipient MHC I and II antibodies) for control, 10-week, and 20-week animals

\begin{tabular}{lcccc}
\hline \multicolumn{1}{c}{ PRA values } & $\begin{array}{c}\text { Baseline } \\
\text { MHC I }\end{array}$ & $\begin{array}{c}\text { Baseline } \\
\text { MHC II }\end{array}$ & $\begin{array}{c}\text { Explant } \\
\text { MHC I }\end{array}$ & $\begin{array}{c}\text { Explant } \\
\text { MHC II }\end{array}$ \\
\hline Control & & & & \\
Sham & $46 \%$ & $0.20 \%$ & $34 \%$ & $0.11 \%$ \\
Sham & $47 \%$ & $0.19 \%$ & $32 \%$ & $0.10 \%$ \\
Fresh & $1.0 \%$ & $1.21 \%$ & $82.3 \%$ & $53.9 \%$ \\
$\quad$ Fresh & $13 \%$ & $0.24 \%$ & $53 \%$ & $32.3 \%$ \\
10-wk studies & & & & \\
Cryopreserved & $26.8 \%$ & $34 \%$ & - & $0.27 \%$ \\
Cryopreserved (mortality) & - & - & $34 \%$ & - \\
Cryopreserved decellularized & $28 \%$ & $0.27 \%$ & $51 \%$ & $0.27 \%$ \\
Cryopreserved decellularized & $43 \%$ & $1.15 \%$ & $27 \%$ & $10 \%$ \\
Fresh decellularized & $21 \%$ & $40 \%$ & $13 \%$ & $0.12 \%$ \\
$\quad$ Fresh decellularized & $29 \%$ & $0.11 \%$ & $3.4 \%$ & $0.13 \%$ \\
20-wk studies & & $1.3 \%$ & $98 \%$ & $0.12 \%$ \\
Cryopreserved & $15 \%$ & $0.40 \%$ & $41 \%$ & $0.48 \%$ \\
Cryopreserved & $0.63 \%$ & $0.63 \%$ & $13.5 \%$ & $2.1 \%$ \\
Cryopreserved decellularized & $5.0 \%$ & $0.14 \%$ & $6.6 \%$ & $0.33 \%$ \\
Cryopreserved decellularized & $4.4 \%$ & $0.10 \%$ & $39.6 \%$ & $0.94 \%$ \\
Fresh decellularized & $10 \%$ & $0.09 \%$ & $0.09 \%$ \\
Fresh decellularized & $68 \%$ & & & \\
\hline
\end{tabular}

Differences in assay results between implant and explant values of greater than $10 \%$ are considered positive for circulating panel reactive antibody (as per human assay manufacturer recommendations). PRA, Panel reactive antibody; $M H C$, major histocompatibility complex.

(fresh) allograft valves were implanted as putative positive controls. These animals demonstrated strong MHC I and II circulating PRA responses.

Cryopreserved and decellularized pulmonary artery tissues were then tested and implanted as patch repairs of defects in both the pulmonary artery and aorta. Levels of circulating antibody were measured before implantation and at 10 -week and 20 -week intervals. Two of the 3 cryopreserved animals receiving patch implants had positive results for circulating antibody response. The increases occurred mainly in the MHC I class. These observations are typical and similar to those observed in the human population after the implantation of cryopreserved tissues. Human studies of MHC I antibody response detected increases about $50 \%$ of the time, and MHC II response occurred $20 \%$ to $30 \%$ of the time. ${ }^{16,17}$ These observations can be attributed to antigen frequency and location. Endothelial cells are rich in MHC I antigen, but their presence can vary as a result of the consequences of cryopreservation and disinfection techniques. ${ }^{18-20}$ Residual endothelial cells rich in MHC I antigen on cryopreserved tissue grafts can allosensitize the host and elicit a measurable antibody response more readily than MHC II antigens located within the tissue matrix. As clinically used, cryopreserved tissues vary unpredictably in retained antigen-rich cells, which likely partially explains the variable durability experiences. ${ }^{21}$

None of the four 10-week animals and only one of the four 20 -week animals with decellularized patches demon- strated a positive PRA antibody response. The one positive response was to MHC I class antigen. The response was also considerably less than its cryopreserved control counterpart. Similar to human studies, there was a measurable and statistically significant difference in circulating PRA levels when comparing decellularized with cryopreserved tissue $(P<.05$, ANOVA).

Immunohistochemistry was performed on samples of all preimplantation tissues to validate the circulating antibody response measurements. The immunohistochemistry targeted MHC I and II antigens. As expected, nonprocessed tissue specimens with histologically intact endothelium expressed large amounts of both MHC I and II antigen, which also provoked the greatest increase in MHC I and II circulating antibodies, as demonstrated by flow cytometry. Cryopreserved tissue patches demonstrated both varying levels of retained endothelial cellularity and subsequent MHC I PRA responses. Decellularized tissue scaffolds (whether preceded by classic cryopreservation or not) demonstrated the smallest detectable amounts of MHC I and II antigen and also provoked little or no PRA response.

In modifying the beaded system, certain differences between the human and sheep models become apparent. Sheep demonstrated increased resting baseline of MHC I values. The most likely factor appears to be bacterial (nonspecific binding from higher levels of circulating immunoglobulin $\mathrm{G}$ generated toward gut flora), which is consistent with known high levels of gastrointestinal flora in ruminants. Increased 
baseline values can be decreased with the administration of antibiotics. The MHC I measured values, however, drift back toward the higher baseline values over time once the antibiotics have been stopped. This problem can be corrected by gating the human negative control to $99.7 \%$. The threshold for positive responses was defined as $10 \%$ over baseline (as recommended by the human test manufacturer) to minimize type 1 error. Small increases in PRA levels soon after implantation might be difficult to identify after increased baseline values. Lagging time-dependent return to baseline, however, appears to identify tissue-specific PRA responses when measured over a longer time period. Such increased baseline values have also been noted in human subjects and are inherent to the nonspecific binding properties of the beads. Antibiotic administration throughout the chronic phase of the experiment is a potential, although it is a costly option and risks interfering with the bacterial component of ruminant food digestion. It is also critical to analyze individual animal baselines with each time-interval specimen. MHC II PRA measurements did not demonstrate this nonspecific binding phenomenon.

Although the PRA response was positive for nonprocessed implants, there was a measurable difference between them. This might be attributed to the fact that the nonprocessed valves were harvested from different animals within our own laboratory, and in doing so, certain HLA similarities might have existed between the donor and the recipient. Although ordered at different times, all sheep were obtained from the same supplier, who maintains a herd of laboratorygrade sheep, and thus inbreeding could have led to more similar genotypes.

Understanding the correlation between allosensitization, HLA incompatibility, and tissue degeneration will be critical for qualifying tissue-engineered constructs that purport to avoid the consequences of recipient immune responses. Our modified ovine method for detecting circulating antibody seems to replicate the results seen in human trials. Such a chronic animal model will be useful in the evaluation of new bioengineering technologies on the basis of biologic products that might retain antigenic proteins. Successfully decellularized scaffolds avoid allosensitization because virtually no MHC antigens are retained. Incomplete decellularization with an excess of cellular debris, however, can provoke significant immune-mediated inflammation, resulting in functional failure. ${ }^{21}$ If residual cytokines remain in the extracellular matrix after detergent-enzyme decellularization (which always results in cell necrosis), they can potentially promote nonspecific inflammatory responses during reperfusion, even in the absence of retained intact cells, exacerbating the scar and foreign-body healing responses, which in turn might promote immune responses and ultimate failure of the tissue-engineered construct. MHC immunohistochemistry staining identifies not only antigenic intact cells but also residual cellular debris. Demonstrations of acellularity with routine staining methods, absence of retained donor DNA in preimplantation samples, or both, are insufficient evidence of adequate reduction of antigenicity by putative decellularization methods.

PRA titers have been traditionally used in the evaluation of transplant recipients because the method assays a generalized humoral response that has been useful in the prediction of chronic cell-mediated rejection when activated clinically (regardless of the specific antigen). Cryopreserved valve transplants seem to fail as a consequence of delayed or long-term cell-mediated chronic rejection. This prolonged failure mode time course is likely the result of lower levels of antigen when compared with those seen in viable solid-organ transplants. However, an early detectable humoral response might be identified and used to predict ultimate T cell- and B cell-mediated pathways of rejection. Thus, despite the generalized or lumped character of the panel of reactive antibodies as a test, this very characteristic specificity has made it sensitive and useful for predicting long-term tolerance for specific transplanted tissues. Tissueengineered constructs, whether derived within or outside of species, need to be tested for such immunogenic potential.

Thus, in this context preclinical animal chronic implant evaluations of bioengineered cardiovascular constructs, such as decellularized allograft scaffolds, should include documentation of the time course of PRA responses to correlate preimplantation treatments with potential allosensitization and its effects on ultimate performance, durability, and even explant morphologic analysis of inflammation, cell repopulation, and tissue healing. This should aid in determining whether a putative scaffold design has actually been adequately biologically decellularized before being recellularized with phenotypically appropriate cells that will regenerate normal tissue structure and function or whether the scaffold material risks provoking immune responses (as differentiated from nonspecific foreign-body responses), leading to donor structural protein destruction with inflammation, followed by fibroblast scar formation. Such a sequence will lead to functional deterioration, loss of durability, and performance characteristics and is a prototypical failure mode exemplified by the variable clinical experience with homograft valves, which must be avoided to achieve the promise of tissue-engineered valves and arteries.

\section{References}

1. Green M, Walsh MD, Dare A, Hogan PG. Histologic and immunohistochemical responses after aortic valve allografts in the rat. Ann Thorac Surg. 1998;66:5216-20.

2. Zhao X, Green M, Frazer IH, Hogan P, O'Brien MF. Donor specific immune response after aortic valve allografting in the rat. Ann Thorac Surg. 1994 57:1158-63.

3. Wagner E, Roy R, Marois Y, Douville Y, Guidoin R. Posttransplant antibodies and fresh venous allograft failure in dogs. Transplantation. 1994;58:537-42. 
4. Hoekstra FM, Witvliet M, Knoop C. Donor-specific anti-human leukocyte antigen class I antibodies after implantation of cardiac valve allografts. J Heart Lung Transplant. 1997;16:570-2.

5. Fischlein T, Schutz A, Haushofer M, et al. Immunologic reaction and viability of cryopreserved homografts. Ann Thorac Surg. 1995; 60(suppl):S122-6.

6. Hawkins JA, Breinholt JP, Lambert LM, et al. Class I and Class II anti-HLA antibodies after implantation of cryopreserved allograft material in pediatric patients. J Thorac Cardiovasc Surg. 2000;119:32430.

7. Lupinetti FM, Cobb S, Kioschos HC, Thompson SA, Walters KS, Moore KC. Effect of immunological differences on rat aortic valve allograft calcification. J Card Surg. 1992;7:65-70.

8. Hogan P, Duplock L, Green M, et al. Human aortic valve allografts elicit a donor-specific immune response. J Thorac Cardiovasc Surg. 1996;112:1260-7.

9. Clarke DR, Campbell DN, Hayward AR, Bishop DA. Degeneration of aortic valve allografts in young recipients. J Thorac Cardiovasc Surg. 1993;105:934-42.

10. Rajani B, Uree RB, Ratliff NB. Evidence for rejection of homograft valves in infants. J Thorac Cardiovasc Surg. 1998;115:111-7.

11. Vogt P, Stallmach T, Niederhauser U, et al. Explanted cryopreserved allografts: a morphological and immunohistochemical comparison between arterial allografts and allograft heart valves from infants and adults. Eur J Cardiothorac Surg. 1999;15:639-45.

12. Shaddy RE, Hunter DD, Osborn KA, et al. Prospective analysis of HLA immunogenicity of cryopreserved valved allografts used in pediatric heart surgery. Circulation. 1996;94:1063-7.
13. Breinholt JP, Hawkins JA, Lambert LM, Fuller TC, Profaizer T, Shaddy RE. A prospective analysis of the immunogenicity of cryopreserved nonvalved allografts used in pediatric heart surgery. Circulation. 2000;102(Suppl III):III179-82.

14. Smith JD, Ogino H, Hunt D, et al. Humoral immune response to human aortic valve homografts. Ann Thorac Surg. 1995;60(Suppl): S127-30.

15. Hawkins JA, Hillman ND, Lambert LM, Jones J, et al. Immunogenicity of decellularized cryopreserved allografts in pediatric cardiac surgery; comparison with standard cryopreserved allografts. J Thorac Cardiovasc Surg. 2003;126:247-52.

16. Hoekstra F, Knoop C, Vaessen L, et al. Donor-specific immune response against human cardiac valve allografts. J Thorac Cardiovasc Surg. 1996 112:281-6.

17. Hoekstra FM, Witvliet M, Knoeep CY, et al. Immunogenic human leukocyte antigen Class II antigens on human cardiac valve induce specific alloantibodies. Ann Thorac Surg. 1998;66:2022-6.

18. Verghese S, Cherian KM. HLA expression in aortic and pulmonary homografts: effects of cryopreservation. Indian Heart. 2002;54:394-8.

19. Lupinetti FM, Tsai TT, Kneebone JM, Bove EL. Effects of cryopreservation on the presence of endothelial cells on human valve allografts. Thorac Cardiovasc Surg. 1993;106:912-7.

20. Lang SJ, Giordano MS, Cardon-Cardo C, et al. Biochemical and cellular characterization of cardiac valve tissue after cryopreservation or antibiotic preservation. J Thorac Cardiovasc Surg. 1994;108:63-7.

21. Hilbert SL. Section VII: allograft heart valves: morphologic, biochemical, and explant pathology studies. In: Hopkins RA, editor. Cardiac reconstructions with allograft tissues. New York: Springer-Verlag; 2004. p. 193-231. 